\title{
Experimental Tests of Discrete Symmetries with Kaons and B-mesons ${ }^{1}$
}

\author{
Bernard Peyaud \\ CEA-Saclay, DAPNIA, Gif sur Yvette 91191 Cedex France
}

\begin{abstract}
During the last decades, experiments using K mesons have provided precise measurements of $\mathrm{CP}$ violation parameters which are important inputs for the understanding of flavour physics. They include the measurements of $\operatorname{Re}\left(\varepsilon^{\prime} / \varepsilon\right)$ by NA48 and $\mathrm{KTeV}$ experiments using $\mathrm{K}_{\mathrm{L}}$ and $\mathrm{K}_{\mathrm{S}}$, the search for $\mathrm{CP}$ violation in $\mathrm{K}^{ \pm} \rightarrow 3 \pi$ decays and rare decay processes allowing to search for new physics and to test chiral perturbation theory. Moreover, NA62, a new experiment at the CERN/SPS, will measure the branching ratio of $\mathrm{K}^{+} \rightarrow \pi^{+} v \bar{v}$ to test further the Standard Model. Experimental efforts in the kaon sector will thus continue for some time. Alternatively, in the B-meson system, there are more decay modes available, and the Standard Model makes precise predictions for $\mathrm{CP}$ violation in a number of these. The B-meson system is therefore a very attractive area to study CP violation and to search for new physics. The LHCb experiment at the Large Hadron Collider (LHC) and its prospects in the study of CP violation in the B-meson sector are discussed.
\end{abstract}

Keywords: $\mathrm{CP}$ violation, $\mathrm{K}$ and $\mathrm{B}$ mesons

PACS: 11.30.Er, 13.25Es, $13.25 \mathrm{Hw}, 14.40 \mathrm{Aq}$

\section{INTRODUCTION}

The discovery of $\mathrm{CP}$ violation was made in the neutral kaon system which continues to be a great resource to study this fundamental phenomenon of Nature. It was quickly established that the dominant part of the process comes from indirect $\mathrm{CP}$ violation where the physical states $\mathrm{K}_{\mathrm{L}}$ and $\mathrm{K}_{\mathrm{S}}$ deviate slightly from the $\mathrm{K}_{2}(\mathrm{CP}=-1)$ and $\mathrm{K}_{1}(\mathrm{CP}=1)$ eigenstates. This mixing is characterized by the complex parameter $\varepsilon \approx 2.3 \cdot 10^{-3} \cdot \exp ^{\mathrm{i} 45^{\circ}}$ which fixes the size of the deviation from pure CP states. Within the Standard Model (SM) $\varepsilon$ is related to the phase $\delta$ of the CKM mixing matrix describing the 3 generations of quarks. There is also a possibility for direct $\mathrm{CP}$ violation in $\mathrm{K}_{2} \rightarrow$ $\pi \pi$ decays and it is fundamental to understand this possible effect within the SM. Direct $\mathrm{CP}$ violation in the kaon system is parameterized by $\varepsilon^{\prime}$ and corresponds to different decay amplitudes for $\mathrm{K}_{0}$ and $\overline{\mathrm{K}}_{0}$. When expressed to first order in terms of $\varepsilon$ and $\varepsilon$ ' the amplitude ratios for neutral decays $\eta_{00} \equiv A\left(K_{L} \rightarrow \pi^{0} \pi^{0}\right) / A\left(K_{S} \rightarrow \pi^{0} \pi^{0}\right)$ and for charged decays $\eta_{+-} \equiv \mathrm{A}\left(\mathrm{K}_{\mathrm{L}} \rightarrow \pi^{+} \pi^{-}\right) / \mathrm{A}\left(\mathrm{K}_{S} \rightarrow \pi^{+} \pi^{-}\right)$are given respectively by $\eta_{00}=\varepsilon-2 \varepsilon^{\prime}$ and by $\eta_{+-}=\varepsilon+\varepsilon^{\prime}$. The measurement of the double ratio $R=\left|\eta_{00} / \eta_{+-}\right|^{2}$ gives access to direct $\mathrm{CP}$ violation through $\mathrm{R}=1-6 \operatorname{Re}\left(\varepsilon^{\prime} / \varepsilon\right)$ which has received a lot of theoretical and experimental investigations.

\footnotetext{
${ }^{1}$ Invited talk presented at the UAE-CERN Workshop, 26-28 November 2007, Al-Ain, United Arab Emirates
} 


\section{THE DISCOVERY OF CP VIOLATION}

In 1964, a remarkable experiment at Brookhaven National Laboratory (BNL) reported evidence of the long-lived neutral kaon decaying in the forbidden state $\pi^{+} \pi^{-}$. Under the assumption of CP symmetry the model [1] based on $\mathrm{K}_{0}$ mixing with $\overline{\mathrm{K}}_{0}$ predicts the long lifetime state $\mathrm{K}_{2}$ with eigenvalue $\mathrm{CP}=-1$ and therefore is not allowed to decay to $2 \pi$ which has $\mathrm{CP}=+1$. In 1956 , the first observation [2] of $\mathrm{K}_{2}$ was made at BNL in a bubble chamber. The typical observation was the allowed mode $\mathrm{K}_{2} \rightarrow \pi^{+} \pi^{-} \pi^{0}$ and also the semileptonic modes $\mathrm{K}_{\mathrm{e} 3}$ and $\mathrm{K}_{13}$. None of 186 observed events could be kinematically fitted under the assumption of $\mathrm{K}_{2} \rightarrow \pi^{+} \pi^{-}$decay and, as expected, CP was conserved to high accuracy. In 1961, a similar bubble chamber experiment [3] conducted at JINR-Dubna reported on 225 decays with no sign of $\mathrm{K}_{2} \rightarrow \pi^{+} \pi^{-}$decay and the conclusion was reached that the branching ratio could not exceed 1/400. Then, in 1963, a liquid hydrogen bubble chamber was placed in a neutral beam at the BNL Cosmotron and $252 \mathrm{~K}_{2}$ decays were observed. These events were analyzed as $\mathrm{K}_{2} \rightarrow \pi^{+} \pi^{-}$decays through their angular distribution where a forward peak of 19 events was given the interpretation of "anomalous regeneration" [4]. This perplexing result was one of the reasons that led the Princeton team in April 1963 to submit a Letter of Intent for an experiment based on an existing apparatus, a doublearmed spectrometer with spark chambers and Cerenkov counters as shown in Figure 1. The BNL Directorate gave agreement in May 1963 and the apparatus was installed and ready on June $2^{\text {nd }}$. The run lasted until the end of July with 70000 triggers taken for regeneration studies, 47000 triggers for CP limit and 23000 triggers to study the "Adair effect" on hydrogen. By looking at mass slices below, above and at the $\mathrm{K}_{0}$ mass a peak in the forward direction was detected during a first analysis made between October and December 1963. The team decided to reanalyze the events with more accuracy in the measurement of the spark positions. From January to April 1964 many attempts were made to "kill" the peak but none was successful. One year after they had submitted their proposal the Princeton group demonstrated to the scientific community that the $\pi^{+} \pi^{-}$decay of the long lived kaon was occurring with a branching ratio of $(2 \pm 0.4) \times 10^{-3}$. This unexpected discovery [5] was "made with a simple apparatus, designed to ask the right question of nature and received a clear response".
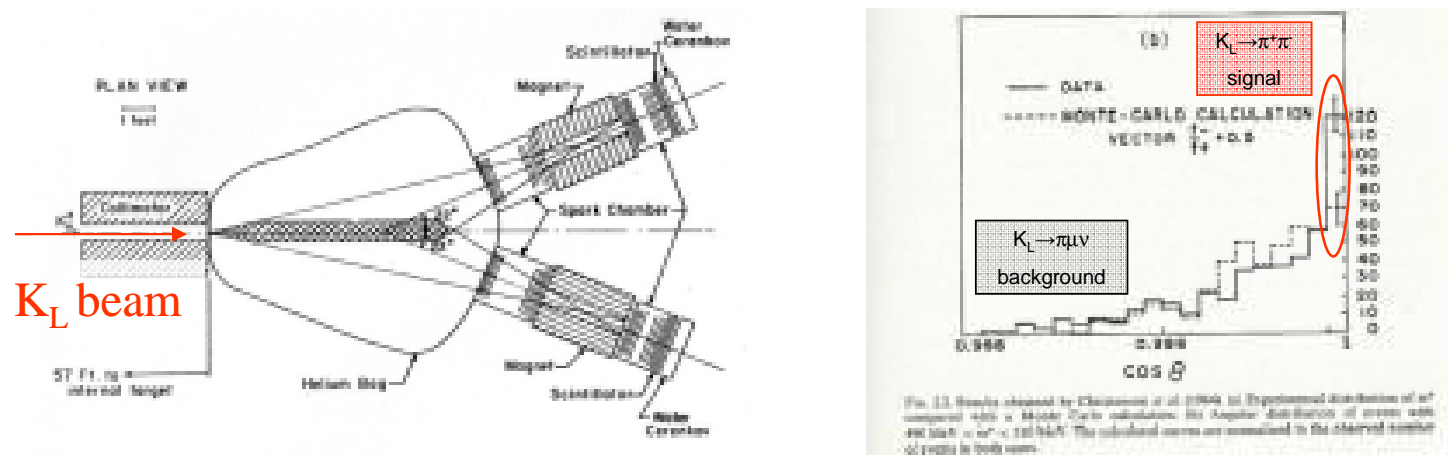

FIGURE 1: Schematics of the experiment with which the Princeton group discovered CP violation at BNL. The peak at $\cos \theta=1$ shows the evidence for $\mathrm{K}_{\mathrm{L}} \rightarrow \pi^{+} \pi^{-}$. 


\section{THE QUEST FOR DIRECT CP VIOLATION}

\section{Super weak theory is wounded by NA31}

If $\varepsilon$ is the only source of $\mathrm{CP}$ violation we would expect $\eta_{00}=\eta_{+-}$. as predicted by the super-weak hypothesis [6]. In 1979 a proposal was made at Fermilab to measure the ratio $\mathrm{R}$ of $\mathrm{CP}$ violating rates $\left|\eta_{00}\right|^{2}$ and $\left|\eta_{+-}\right|^{2}$ to a precision better that $1 \%$. The E617 experiment achieved 3\% accuracy only but paved the way for the next measurement made by E731. The principle of the experiment relies on the use of two neutral beams with one of them incident on a regenerator (Figure 2a) thus allowing simultaneous study of $\mathrm{K}_{\mathrm{S}} / \mathrm{K}_{\mathrm{L}}$ decay products in the apparatus. The most important parts of the detector are drift chambers to measure the momenta of charged particles, lead-glass electromagnetic calorimeter to measure $\gamma$ 's from $\pi^{0}$ and muon hodoscope. Another proposal was made in 1980 at CERN with a different principle where neutral and charged rates are measured simultaneously in the NA31 detector which consist of proportional chambers to measure the charged directions, liquid argon photon calorimeter, hadron calorimeter and muon veto. The simultaneous $\mathrm{K}_{\mathrm{S}}$ and $\mathrm{K}_{\mathrm{L}}$ beams are obtained by using a movable target (Figure $2 \mathrm{a}$ ) inside a long vacuum tunnel. Both detectors use high performance electromagnetic calorimeters (i.e. good $\gamma$ position and energy resolution) in order to reject the main background from $3 \pi^{0}$ decays in the neutral mode. In the charged mode E731 achieves good background rejection of $\mathrm{K}_{\mathrm{e} 3}$ by combining momentum measurement in the drift chamber spectrometer and energy measurement in the electro-magnetic calorimeter (E/p identification of electron) whereas NA31 obtains good $\pi$ identification with the hadronic calorimeter.
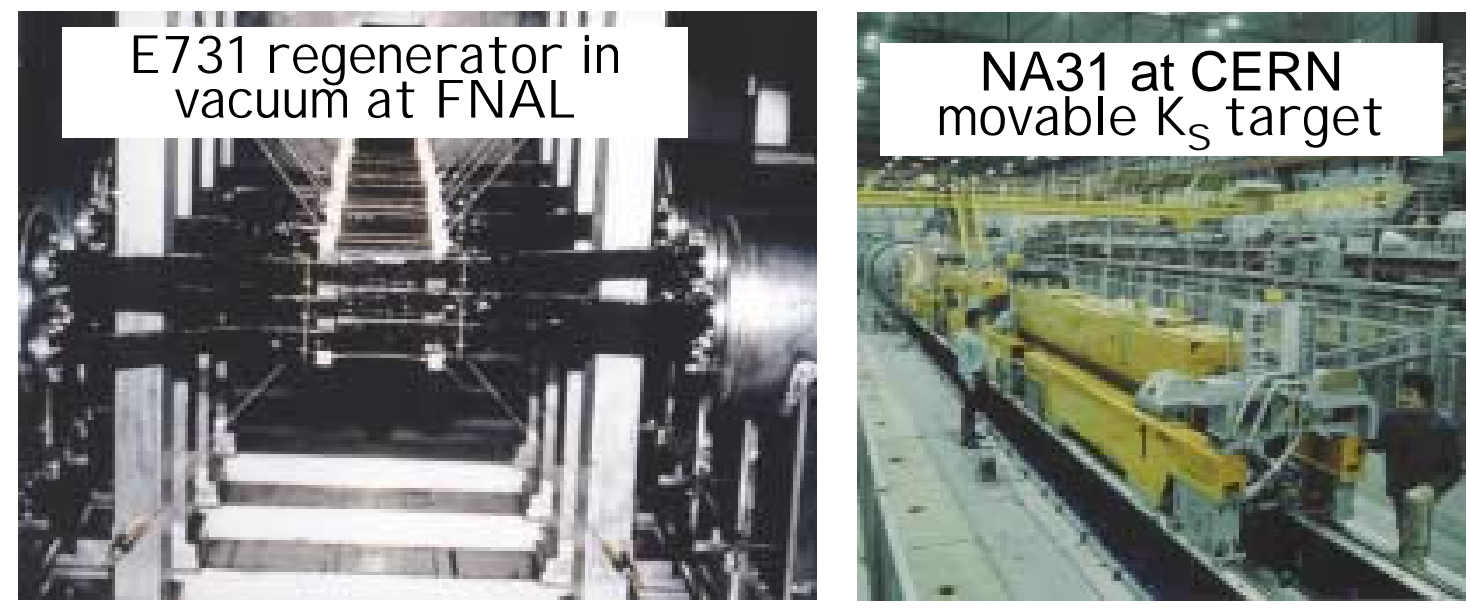

FIGURE 2: a) The E71 carbon regenerator used at FNAL and b) the NA31 $\mathrm{K}_{\mathrm{S}}$ target at CERN.

At the concluding talk of the Blois Conference in 1989 the status of the analysis of the two experiments was the following:

NA31

$\operatorname{Re}\left(\varepsilon^{\prime} / \varepsilon\right)=(33 \pm 11) \times 10^{-4}$,

E731 (very preliminary)

$$
\operatorname{Re}\left(\varepsilon^{\prime} / \varepsilon\right)=(-10 \text { to } 10 \pm 15) \times 10^{-4},
$$


The evidence of a non-zero effect was to some extent diluted by the variance between the results of NA31 and E731 and it was concluded that the experiments must be continued to still higher accuracy.

The magnitude of direct CP violation signal was still contentious when the analysis of the two experiments [7] , [8] were completed in 1996 with the final results:

$$
\begin{array}{ll}
\text { NA31 } & \operatorname{Re}\left(\varepsilon^{\prime} / \varepsilon\right)=(23 \pm 6.5) \times 10^{-4}, \\
\text { E731 } & \operatorname{Re}\left(\varepsilon^{\prime} / \varepsilon\right)=(7.4 \pm 6.5) \times 10^{-4} .
\end{array}
$$

The next round of experiments on $\operatorname{Re}\left(\varepsilon^{\prime} / \varepsilon\right)$ had already started in the early 90's at CERN with NA48 and at FNAL with KTeV.

\section{The experimental fix of $\varepsilon^{\prime} / \varepsilon$}

The NA48 and KTeV experiments were designed to measure $\operatorname{Re}\left(\varepsilon^{\prime} / \varepsilon\right)$ with an accuracy of $\leq 2 \times 10^{-4}$. In order to reach this requirement the limiting mode $K_{L} \rightarrow \pi^{0} \pi^{0}$ must contain at least 4 million events and the systematic errors be kept at very small levels.

The principle of the NA48 experiment is to use almost collinear $\mathrm{K}_{\mathrm{L}}$ and $\mathrm{K}_{\mathrm{S}}$ beams pointing at the centre of the detector and having similar momentum spectra $\left(\left\langle\mathrm{P}_{\mathrm{K}}\right\rangle=110 \mathrm{GeV} / \mathrm{c}\right)$ and to detect all 4 decay modes at the same time. Large statistics require high-intensity beams and therefore high-rate capability and performance for the detector elements. The identification of the neutral/charged mode is made with high-resolution photon-calorimeter/spectrometer and $\mathrm{K}_{\mathrm{L}}$ and $\mathrm{K}_{\mathrm{S}}$ are separated by tagging. By applying a lifetime weighting procedure to the $\mathrm{K}_{\mathrm{L}}$ decays in both modes one reduces almost completely the acceptance differences with $K_{S}$ due to their lifetimes. The weighted vertex distributions of $\mathrm{K}_{\mathrm{L}}$ are made similar to the $\mathrm{K}_{\mathrm{S}}$ ones thus bringing the experiment close to "gedanken" situation with $\mathrm{K}_{\mathrm{L}} / \mathrm{K}_{\mathrm{S}}$ coming from the same source.
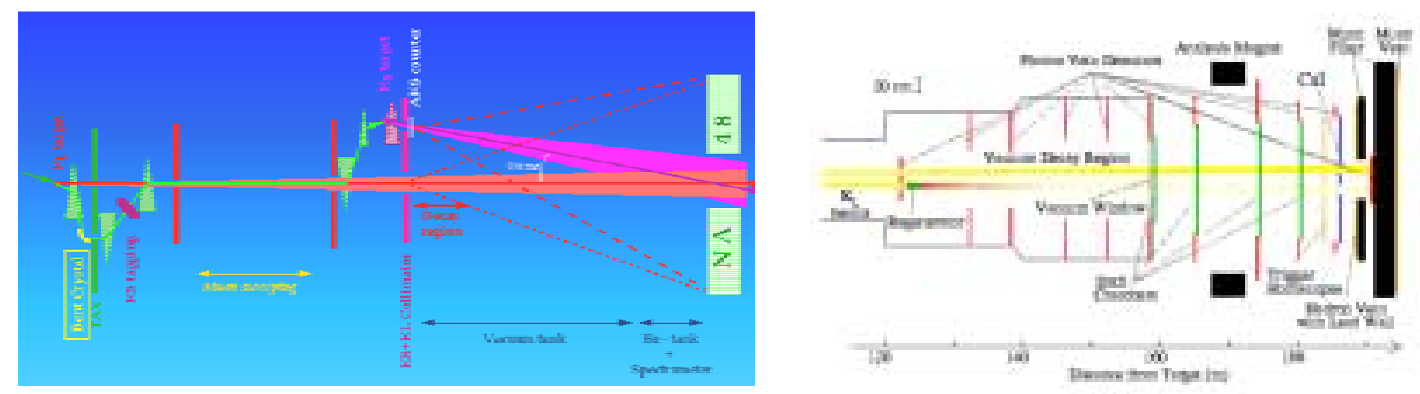

FIGURE 3: $\quad$ a) the $\mathrm{K}_{\mathrm{L}} / \mathrm{K}_{\mathrm{S}}$ beam line used at CERN by NA48

b) the $\mathrm{K}_{\mathrm{L}}$ and regenerated $\mathrm{K}_{\mathrm{S}}$ used at FNAL by $\mathrm{KTeV}$.

A schematic view of the neutral kaon beam lines and of the detector layout is shown in Figure 3a. The decay region extends over $90 \mathrm{~m}$ inside a vacuum tank terminated by a thin Kevlar window. It is followed by the detector elements installed between $216 \mathrm{~m}$ to $255 \mathrm{~m}$ downstream of the $\mathrm{K}_{\mathrm{L}}$ target. A magnetic spectrometer is inside a helium tank and is followed by a hodoscope to trigger on the charged mode, a liquid krypton $(\mathrm{LKr})$ calorimeter, a hadronic calorimeter, and a muon detector. 
The principle of the $\mathrm{KTeV}$ experiment is to use a double $\mathrm{K}_{\mathrm{L}}$ beam and to produce $K_{S}$ by regeneration of one of them as shown in Figure $3 b$. The state of the art detector is a photon calorimeter made of pure CsI crystals. The decay region is shorter here owing to the fact that kaons are less energetic $\left(\left\langle\mathrm{P}_{\mathrm{K}}\right\rangle=70 \mathrm{GeV} / \mathrm{c}\right)$. For both detectors the mass resolution for $\mathrm{K}_{\mathrm{L}, \mathrm{S}} \rightarrow \pi^{+} \pi^{-}$decays is $\sim 2 \mathrm{MeV} / \mathrm{c}^{2}$ and the time resolution of the hodoscopes is $\sim 200 \mathrm{ps}$.

Direct CP violation has been measured [9] , [10] by both experiments (Figure 4a) and $\operatorname{Re}\left(\varepsilon^{\prime} / \varepsilon\right)$ is now known to be $(16.3 \pm 2.2) \cdot 10^{-4}$. This non-zero value established with 7 standard deviations provides a second number to describe $\mathrm{CP}$ violation in the neutral kaon system. It translates in the matter antimatter asymmetry ratio:

$$
\frac{\Gamma\left(K^{0} \rightarrow \pi^{+} \pi^{-}\right)-\Gamma\left(\bar{K}^{0} \rightarrow \pi^{+} \pi^{-}\right)}{\Gamma\left(K^{0} \rightarrow \pi^{+} \pi^{-}\right)+\Gamma\left(\bar{K}^{0} \rightarrow \pi^{+} \pi^{-}\right)}=(5.04 \pm 0.82) \times 10^{-6}
$$

These successes have been made possible by a remarkable sequence of ambitious experimental programs in Europe and USA as summarized in Table1.

TABLE 1. Data statistics of experiments at FNAL and CERN

\begin{tabular}{lcccc}
\hline Year & experiment & $\mathbf{K}_{\mathbf{L}} \rightarrow \mathbf{2} \boldsymbol{\pi}$ & Background & Data volume \\
\hline 1985 & E617 FNAL & 3,152 & $\sim 11.3 \%$ & $10 \mathrm{~GB}$ \\
1988 & E731 test FNAL & 6,950 & $\sim 5 \%$ & \\
1988 & NA31 CERN & 109,000 & $\sim 4 \%$ & \\
1990 & E731A FNAL & 52,000 & $\sim 5 \%$ & \\
1993 & E731 Final FNAL & 410,326 & $\sim 5 \%$ & $100 \mathrm{~GB}$ \\
1993 & NA31 Final CERN & 428,000 & $\sim 2.7 \%$ & \\
2001 & KTeV FNAL & $10,000,000$ & $\sim 0.48 \%$ & \\
2001 & NA48 CERN & $5,000,000$ & $\sim .16 \%$ & $100 \mathrm{~TB}$ \\
\hline
\end{tabular}

On the theoretical side $\varepsilon ' / \varepsilon$ is very hard to calculate because the sensitivity is in the sizes and phases of similar amplitudes [11] There is agreement that direct CP violation effect is much smaller than the indirect one. Despite huge efforts, $\varepsilon^{\prime} / \varepsilon$ is not yet computed reliably but improvements are expected from lattice calculation

\section{Charged kaons: another attack by NA48}

While at very small level, direct $\mathrm{CP}$ violation is also expected for charged kaon decays in the standard model, and NA48 has used intense $\mathrm{K}^{ \pm}$beams in 2003/04 to measure the Dalitz plot properties of the $\pi^{ \pm} \pi^{+} \pi^{-}$and $\pi^{ \pm} \pi^{0} \pi^{0}$ decays modes. If CP invariance holds strictly the matrix elements $|\mathrm{M}(\mathrm{u}, \mathrm{v})|^{2} \propto 1+\mathrm{g}^{ \pm} \mathrm{u}+\mathrm{hu}^{2}+\mathrm{kv}^{2}$ for $\mathrm{K}^{ \pm}$ must be identical with $\mathrm{g}^{+}=\mathrm{g}^{-}$whereas the SM asymmetry $\mathrm{A}_{\mathrm{g}}=\left(\mathrm{g}^{+}-\mathrm{g}^{-}\right) /\left(\mathrm{g}^{+}+\mathrm{g}^{-}\right)$is expected to be in the range $\left(10^{-5}\right.$ to $\left.10^{-4}\right)$. This precision measurement [12] was done by NA48 with final samples of $3.1 \times 10^{9} \mathrm{~K}^{ \pm} \rightarrow \pi^{ \pm} \pi^{+} \pi^{-}$decays and $91 \times 10^{6} \mathrm{~K}^{ \pm} \rightarrow \pi^{ \pm} \pi^{0} \pi^{0}$ decays giving respectively the following results [13]:

$$
\mathrm{A}_{\mathrm{g}}\left(\pi^{ \pm} \pi^{+} \pi^{-}\right)=(-1.5 \pm 2.1) \cdot 10^{-4} \text { and } \mathrm{A}_{\mathrm{g}}\left(\pi^{ \pm} \pi^{0} \pi^{0}\right)=(-1.5 \pm 2.1) \times 10^{-4}
$$


Compared to previous experiments these results are 10 times more accurate and the errors are dominated by statistics. No CP violation was found but the window to search for new physics has been reduced dramatically (Figure 4b). J. Cronin's hope of 1980 "that at some epoch, perhaps distant, this cryptic message from Nature will be deciphered" has been significantly but partially satisfied.

Another important exploration of $\mathrm{CP}$ violation is being prepared by experiment NA62 at CERN [14]. This is the search for the very rare decay $\mathrm{K}^{+} \rightarrow \pi^{+} v \bar{v}$ which is a CKM suppressed process occurring through Flavour Changing Neutral Current. It is very clean theoretically since short distance contributions are dominant and hadronic matrix elements are obtained from the measured decay $\mathrm{K}^{+} \rightarrow \pi^{0} \mathrm{l} v$. The SM prediction of the branching ratio is $\mathrm{BR}\left(\mathrm{K}^{+} \rightarrow \pi^{+} v \overline{\mathrm{v}}\right)=(8.0 \pm 1.1) \times 10^{-11}$ for which the experimental measurement made by E787/949 is $\mathrm{BR}\left(\mathrm{K}^{+} \rightarrow \pi^{+} v \bar{v}\right)=\left(1.47^{+1.30}{ }_{-0.89}\right) \times 10^{-10}$ (3 events).

The NA62 experiment aims at observing $\mathrm{O}(100) \mathrm{K}^{+} \rightarrow \pi^{+} v \bar{v}$ events with a background level not exceeding $10 \%$. The sensitivity to new physics is again the driving force of this enterprise.
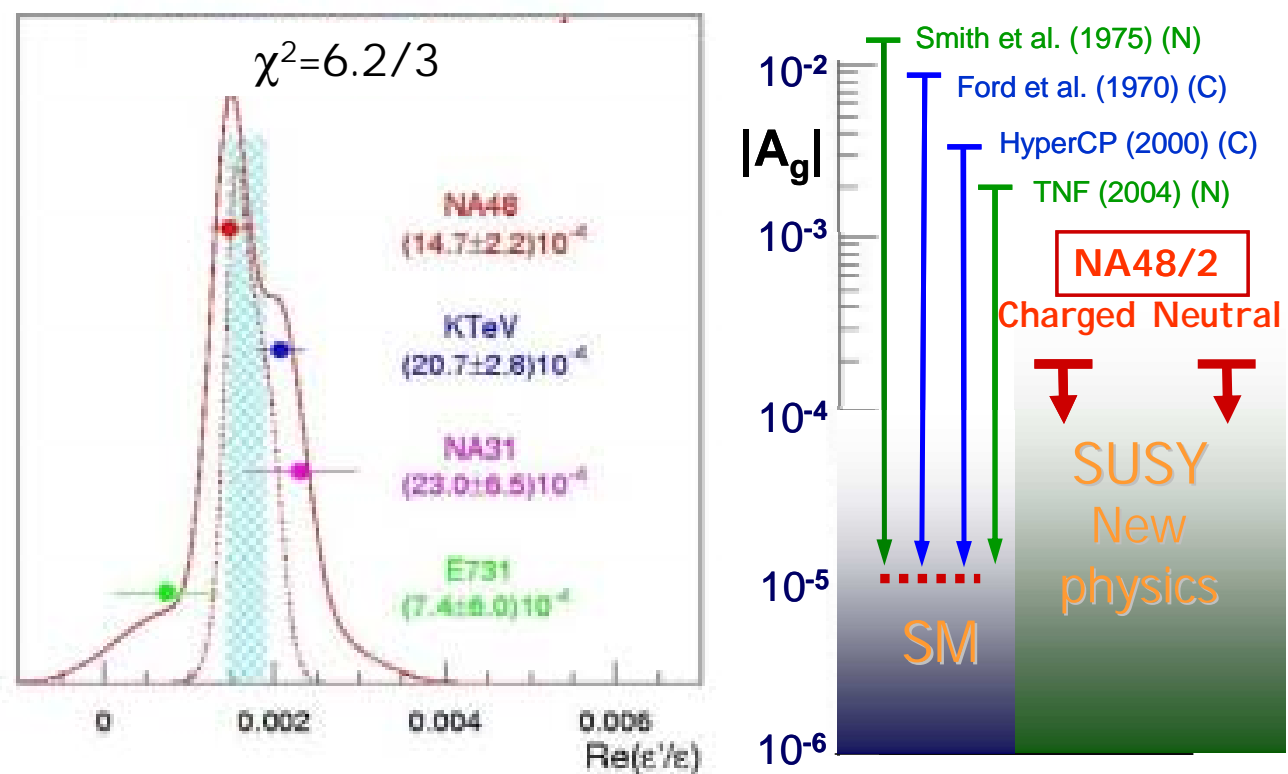

FIGURE 4: $\quad$ a) Direct $\mathrm{CP}$ violation measurements of $\operatorname{Re}\left(\varepsilon^{\prime} / \varepsilon\right)$

b) Limits on $\mathrm{Ag}$ from $\mathrm{K}^{ \pm}$asymmetry measurements.

\section{THE USE OF B MESONS}

Neutral kaon mixing has played a major role in understanding how the $\mathrm{CP}$ violating phase $\delta$ enters the SM. Similarly Bd mixing was the first experimental phenomenon to correctly anticipate the large value of the top quark mass and later the precise measurements of many different B-meson decay modes obtained in the B-factories, allowed the CKM picture of mixing and $\mathrm{CP}$ violation to be verified at the few per cent level. The unitarity triangle (Figure 5a) is known today with good accuracy from the 
measurement of $|\mathrm{Vcb} / \mathrm{Vub}|, \Delta m\left(\mathrm{~B}_{\mathrm{d}}\right)$, and the observation on $\Delta m\left(\mathrm{~B}_{\mathrm{s}}\right)$. While these quantities are $\mathrm{CP}$-conserving, they imply non-zero angles of the triangle, and therefore $\mathrm{CP}$ violation.
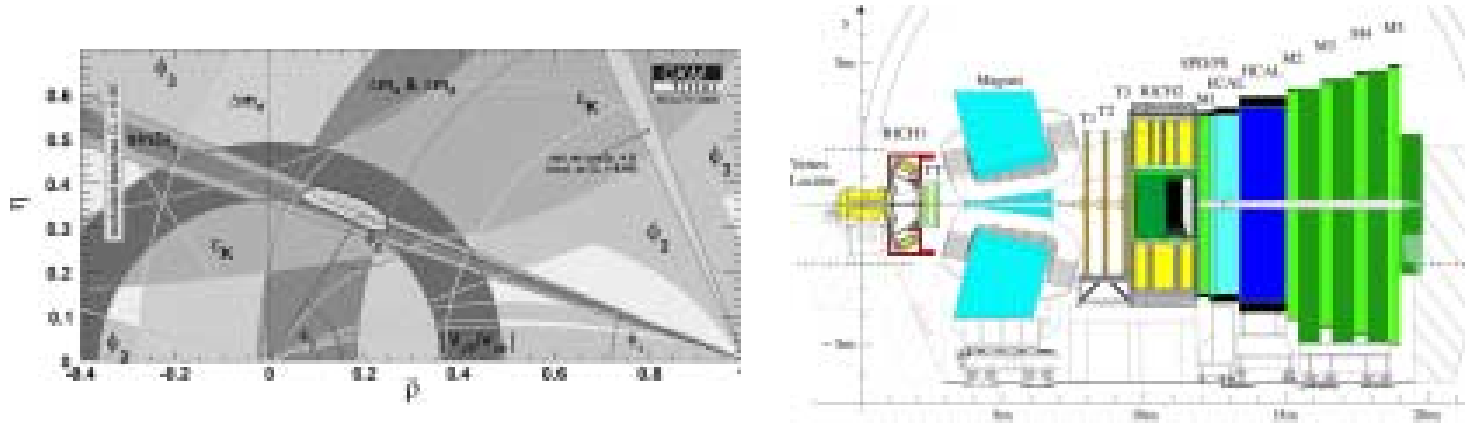

FIGURE 5: $\quad$ a) The impact of $B$ physics on the unitarity triangle

b) The LHCb detector dedicated to B physics at LHC.

The LHCb [15] experiment (Figure 5b) will soon complement and extend the studies made at $\mathrm{B}$ factories. This unique detector will probe $\mathrm{CP}$ violation in the D0 system at the $10^{-3}$ level and flavour physics in the quark sector will continue to be an important research direction. Among the key elements of the detector are the $\mathrm{Si}$ vertex detector that will measure charged particle tracks, and the RICH detectors, that will identify particles. The shopping list of LHCb includes:

- $\quad B d$ and Bs mixing effects (including CPV) in tree decays

- $\quad C P V$ in pure hadronic $b \rightarrow s$ penguin transitions

- Direct $C P V$ (angle $\gamma$ ) in $B \rightarrow D K$ tree decays

- $\quad C P V$ in $B \rightarrow h+h-$ decays (trees + penguins)

- $\quad C P V$ (angle $\alpha$ ) in $B \rightarrow \rho \pi$, $\rho \rho$ decays (trees + penguins)

- $\quad b \rightarrow s \gamma$ and $b \rightarrow s l+l-$ transitions (electromagnetic penguins)

- Pure leptonic B decays

- Charm and charmonium

These measurements will significantly improve the accuracy of the unitarity triangle. With high luminosities LHCb may well succeed in finding decays with rates above the SM predictions thus pointing to phenomena requiring new physics.

\section{CONCLUSIONS}

The non-invariance of the fundamental interactions under the discrete symmetries $\mathrm{C}, \mathrm{P}$ and T has played a central role in the development of the SM. Quantitatively, the measurements of $\mathrm{CP}$ violation in several channels, both with kaons and b mesons, is perfectly consistent with the SM, and in particular with one single complex phase $\delta$ as the dominant source of $\mathrm{CP}$ violation in the quark sector.

The measurement of very rare FCNC decays such as $\mathrm{K}_{\mathrm{L}}^{0} \rightarrow \pi^{0} v \bar{v}, \mathrm{~K}^{+} \rightarrow \pi^{+} v \bar{v}$, or $\mathrm{B}_{\mathrm{d}, \mathrm{s}} \rightarrow l^{+} l^{-}$, the detection of new CP-violating phases in heavy-flavour decays, will undoubtedly provide precious information on the effect of these new phenomena on low-energy physics, and perhaps give important constraints on yet unobserved heavy particles. 


\section{ACKNOWLEDGMENTS}

It was a pleasure to participate to this first UAE-CERN workshop held in Al-Ain in order to help this region of the word deciding upon how to organize their participation in the adventure of finding things yet unknown to mankind. I thank the organizers for their wonderful effort and the invaluable help and kindness that they provided to their guests.

\section{REFERENCES}

1. M. Gell-Mann and A. Pais, Phys. Rev. 97, 1387, (1955).

2. K. Landé et al., Phys Rev. 103, 1901 (1956).

3. D. Neagu et al., Phys Rev. Lett. 6, 552 (1961).

4. L. B. Leipuner et al., Phys Rev. 132, 2285 (1963).

5. J. H. Christenson, J. W. Cronin, V.L. Fitch and R. Turlay, Phys Rev Lett. 13, 138 (1964).

6 L. Wolfenstein, Phys. Rev. Lett. 13, 562, (1963).

7 G. D. Barr et al., Phys. Lett. B 317, 233, (1993).

8 L. K. Gibbons et al., Phys.Rev. Lett. 70, 1203, (1993).

9 A. Alavi-Harati et al., Phys. Rev. D67, 012005 (2003).

10 J. R. Batley et al., Phys. Lett. B 544, 97, (2002).

11 Selected theory papers: http://www.phy.bnl.gov/e949/papers/theory.html.

12 S. Balev for NA48 Collaboration., Rencontres de la Vallée d'Aoste, La Thuile. (2006),

13 J. R. Batley et al., Phys. Lett. B 638, 22, (2006).

14 Proposal to measure the rare decay $\mathrm{K}^{+} \rightarrow \pi^{+} v \bar{v}$ at the CERN SPS, CERN-SPSC-2005-013, SPSC326.

15 LHCb Home page: http://lhcb.web.cern.ch/lhcb 